\title{
CORRIGENDUM
}

\section{Proposal of new strategy for ambulatory blood pressure profile-based management of resistant hypertension in the era of renal denervation}

\author{
Kazuomi Kario
}

Hypertension Research (2013) 36, 564; doi:10.1038/hr.2013.40

Correction to: Hypertension Research (2013) 36, 478-484; doi:10.1038/hr.2013.19; published online 21 March 2013

Since the publication of this article, the errors have been found in Figures 1-3. The errors have now been rectified, and the correct article appears in this issue. The html and online pdf versions have also been rectified. 